\title{
PL SHEAVES AND THEIR CHARACTERISTIC CLASSES
}

\author{
BY HOWARD OSBORN
}

Communicated by S. S. Chern, January 31, 1972

\begin{abstract}
Any PL manifold possesses a natural structure sheaf and a derivation into a sheaf $\mathscr{E}$ of differential forms, from which one obtains the smoothable function algebra and de Rham complex of [1] via global sections. The sheaves $\mathscr{E}$ are part of a fibered category of sheaves $\mathscr{F}$ of modules over PL structure sheaves, into which the classical category of (sheaves of local sections of) vector bundles embeds as a full subcategory. There is a Chern-Weil construction of real characteristic classes which assigns Chern classes to complex sheaves $\mathscr{F}$ and Euler classes to real oriented sheaves $\mathscr{F}$ in such a way that all the usual axioms are satisfied. These classes are precisely the usual real Chern and Euler classes on the subcategory of vector bundles.

In this note we present definitions and statements of some of the main results concerning PL sheaves and their real characteristic classes. The details will appear in [2].
\end{abstract}

1. Antesheaves. The data one needs to describe certain sheaves on PL manifolds arise most naturally in the form of antesheaves, defined in this section. The definition presented here is a slight variant of the definition used in [2]; the resulting sheaves are the same, however.

For any positive integer $p \leqq n$ an open $p$-simplex of $R^{n}$ at 0 is the set of points $x_{1} e_{1}+\cdots+x_{p} e_{p}$ for fixed linearly independent vectors $e_{1}, \ldots, e_{p} \in R^{n}$ and all positive $x_{1}, \ldots, x_{p}$; the open 0 -simplex of $R^{n}$ at 0 is $\{0\}$. A simplicial decomposition of $R^{n}$ at 0 is any decomposition of $R^{n}$ into a disjoint union of finitely many open $p$-simplexes, such that each of the $2^{p}-1$ open simplexes occurring as faces of any open $p$-simplex in the decomposition also belongs to the decomposition. By translation one defines simplicial decompositions of $R^{n}$ at any point $P \in R^{n}$. Finally, if $U$ is any open set of $R^{n}$ a simplicial decomposition $\alpha$ of $U$ at $P \in U$ consists of the intersections with $U$ of the open $p$-simplexes of a simplicial decomposition of $R^{n}$ at $P$.

For any simplicial decomposition $\alpha$ of $U$ at $P \in U$ and any $Q \in U$ the open star set of $Q$ in $\alpha$ is the union of those open simplexes of $\alpha$ whose closures contain $Q$.

Now suppose that an $n$-dimensional PL manifold $M$ is described by an atlas $\left\{\left(U_{i}, \Psi_{i}\right)\right\}$, where $\left\{U_{i}\right\}$ is an open cover of $M$, and where each $\Psi_{i}$ is a homeomorphism of the corresponding $U_{i}$ onto an open set

AMS 1970 subject classifications. Primary 57C50; Secondary 57D20, 58C05.

Key words and phrases. PL sheaves, Chern-Weil construction, Chern classes, Euler classes. 
$\Psi_{i}\left(U_{i}\right) \subset R^{n}$ such that $\Psi_{i} \circ \Psi_{j}^{-1}: \Psi_{j}\left(U_{i} \cap U_{j}\right) \rightarrow \Psi_{i}\left(U_{i} \cap U_{j}\right)$ is a PL homeomorphism whenever $U_{i} \cap U_{j} \neq \varnothing$. Then for any $Q \in U_{i} \cap U_{j}$ there is a neighborhood $V \subset U_{i} \cap U_{j}$ of $Q$ such that the restriction of $\Psi_{i} \circ \Psi_{j}^{-1}$ to $\Psi_{j}(V)$ is linear on the open $p$-simplexes of at least one simplicial decomposition $\beta$ of $\Psi_{j}(V)$ at $\Psi_{j}(Q), p=0, \ldots, n$; it follows for any simplicial decomposition $\gamma$ of $\Psi_{j}(V)$ at $\Psi_{j}(Q)$ which refines $\beta$ that the restriction of $\Psi_{i} \circ \Psi_{j}^{-1}$ to $\Psi_{j}(V)$ is also linear on the open simplexes of $\gamma$.

Let $\mathscr{T}(M)$ denote the set of 4-tuples $(P, U, i, \alpha)$, where $U$ is an open subset of some $U_{i}$ occurring in the atlas $\left\{\left(U_{i}, \Psi_{i}\right)\right\}$ of a given PL manifold $M$, where $P \in U$, and where $\alpha$ is a simplicial decomposition of $\Psi_{i}(U)$ at $\Psi_{i}(P)$.

Lemma. For any atlas $\left\{\left(U_{i}, \Psi_{i}\right)\right\}$ of a $P L$ manifold $M$ the set $\mathscr{T}(M)$ is partially ordered by the relation $(P, U, i, \alpha)<(Q, V, j, \beta)$ which requires that (i) $V \subset U$, (ii) the restriction of $\Psi_{i} \circ \Psi_{j}^{-1}$ to $\Psi_{j}(V)$ is linear on each simplex of $\beta$, (iii) $\Psi_{i}(V)$ is contained in the open star set of $\Psi_{i}(Q)$ in $\alpha$, (iv) $\Psi_{i} \circ \Psi_{j}^{-1}(\beta)$ refines the restriction of $\alpha$ to $\Psi_{i}(V)$, and (v) $\beta$ has at least one more open $(n-1)$-simplex than $\alpha$.

We regard the partially ordered set $\{\mathscr{T}(M),<\}$ as a category with morphisms $(Q, V, j, \beta)>(P, U, i, \alpha)$; this is analogous to the customary category of open sets with inclusions $V \subset U$ as morphisms. A full subcategory of $\{\mathscr{T}(M),<\}$ is cofinal if and only if for each $(P, U, i, \alpha) \in \mathscr{T}(M)$ and each $Q \in U$ there is an object $(Q, V, j, \beta)$ in the subcategory such that $(Q, V, j, \beta)>(P, U, i, \alpha)$.

Definition. An antesheaf on a PL manifold $M$ with atlas $\left\{\left(U_{i}, \Psi_{i}\right)\right\}$ is any contravariant functor on some cofinal subcategory of $\{\mathscr{T}(M),<\}$.

For fixed $P \in M$ it is clear that the subset of those $(P, U, i, \alpha)$ with the given $P$ is directed by the partial ordering " $<$ ". Hence for any antesheaf $\left\{A_{P, U, i, \alpha}, \rho(Q, V, j, \beta ; P, U, i, \alpha)\right\}$ on $M$ one can form the direct limits $A_{P}=\lim A_{P, U, i, \alpha}$ by fixing $P$. For any $(P, U, i, \alpha) \in \mathscr{T}(M)$ and any $Q \in U$ we let $\rho(Q ; P, U, i, \alpha): A_{P, U, i, \alpha} \rightarrow A_{Q}$ denote the inclusion map. The objects $A_{P, U, i, \alpha}$ will always be sets.

Lemma. For any antesheaf $\left\{A_{P, U, i, \alpha}, \rho(Q, V, j, \beta ; P, U, i, \alpha)\right\}$ on a $P L$ manifold $M$ let $\mathscr{S}=\bigcup_{P \in M} A_{P}$, let $\pi: \mathscr{S} \rightarrow M$ carry $A_{P}$ into $P$, and for any $f \in A_{P, U, i, \alpha}$ let $\rho_{f} \subset \pi^{-1}(U)$ consist of the images of $f$ under $\{\rho(Q ; P, U, i, \alpha) \mid Q \in U\} ;$ then $(\mathscr{S}, \pi, M)$ is a sheaf over $M$, the sets $\rho_{f}$ forming a basis of the open sets of $\mathscr{S}$.

2. PL sheaves. We first describe the antesheaf for which the preceding lemma provides the structure sheaf of a PL manifold $M$. 
Define a diffeomorphism $\varphi: R^{+} \rightarrow R^{+}$by setting $\varphi(x)=\exp \sinh \ln x$, and observe that $\lim _{x \rightarrow 0} \varphi^{(q)}(x)=0$ for all $q \geqq 0$. Let $\varphi_{N}$ denote the $N$-fold composition $\varphi \circ \ldots \circ \varphi$, and for any simplicial decomposition $\alpha$ of $R^{n}$ at 0 define $\Phi_{\alpha}: R^{n} \rightarrow R^{n}$ by setting $\Phi_{\alpha}\left(x_{1}, \ldots, x_{p}\right)=\left(\varphi_{N}\left(x_{1}\right), \ldots\right.$, $\left.\varphi_{N}\left(x_{p}\right)\right)$ for the coordinates $x_{1}, \ldots, x_{p}$ of each open $p$-simplex of $\alpha$, where $N$ is the number of open $(n-1)$-simplexes in $\alpha$. (In [1], $N$ represented the number of open 1-simplexes in $\alpha$. The change to the present definition of $N$ does not affect the constructions reported in [1], however, and it is essential for the naturality of antesheaves with respect to PL maps. Naturality will be discussed in [2].) One verifies that $\Phi_{\alpha}$ is a smooth homeomorphism; its inverse is definitely not smooth. More generally, for any simplicial decomposition $\alpha$ of $U \subset R^{n}$ at $P \in U$ one defines a smooth homeomorphism $\Phi_{\alpha}: U \rightarrow \Phi_{\alpha}(U)$ in the same fashion.

Let $M$ be a PL manifold with atlas $\left\{\left(U_{i}, \Psi_{i}\right)\right\}$, and for each $(P, U, i, \alpha)$ $\in \mathscr{T}(M)$ let $A_{P, U, i, \alpha}$ denote the algebra of those functions $f: U \rightarrow R$ such that $f \circ \Psi_{i}^{-1} \circ \Phi_{\alpha}: \Phi_{\alpha}^{-1}\left(\Psi_{i}(U)\right) \rightarrow R$ is smooth. By a tedious verification one shows that if $f \in A_{P, U, i, \alpha}$ and $(P, U, i, \alpha)<(Q, V, j, \beta)$ then $f \mid V \in A_{Q, V, j, \beta}$; hence there is an antesheaf $\left\{A_{P, U, i, \alpha}, \rho(Q, V, j, \beta ; P, U, i, \alpha)\right\}$ in which each $\rho(Q, V, j, \beta ; P, U, i, \alpha)$ is merely the restriction map $f \mapsto f \mid V$; the resulting sheaf is the structure sheaf $\mathscr{A}(M)$ of $M$. One easily verifies that $\Gamma(M, \mathscr{A}(M))$ is the smoothable function algebra $A(M)$ described in [1].

Each $A_{P, U, i, \alpha}$ is isomorphic to a smooth function algebra $C^{\infty}\left(\Phi_{\alpha}^{-1}\left(\Psi_{i}(U)\right)\right)$, so that there are obvious derivations $d_{P, U, i, \alpha}: A_{P, U, i, \alpha} \rightarrow E_{P, U, i, \alpha}$ and homomorphisms $\sigma(Q, V, j, \beta ; P, U, i, \alpha)$ for which $\left\{E_{P, U, i, \alpha}, \sigma(Q, V, j, \beta ; P, U, i, \alpha)\right\}$ is also an antesheaf. The resulting sheaf $\mathscr{E}(M)$ of differential forms admits a derivation $d: \mathscr{A}(M) \rightarrow \mathscr{E}(M)$, and by setting $E(M)=\Gamma(M, \mathscr{E}(M))$ one obtains the de Rham complex $\left(\Lambda_{A(M)} E(M), d\right)$ of [1], for which the de Rham theorem is valid.

The homomorphisms $\sigma$ appearing above are definitely not themselves restriction maps; in fact $\operatorname{det} \sigma(Q, V, j, \beta ; P, U, i, \alpha)$ is an element of $A_{Q, V, j, \beta}$ which vanishes on the image under $\Psi_{j}^{-1}$ of the $(n-1)$-skeleton of $\beta$. Now for any $(R, W, k, \gamma) \in \mathscr{T}(M)$ let $S_{R, W, k, \gamma}$ denote the multiplicative subset of $A_{R, W, k, y}$ generated by the restrictions to $W$ of elements $\operatorname{det} \sigma(Q, V, j, \beta ; P, U, i, \alpha)$, where both $(P, U, i, \alpha)<(Q, V, j, \beta)$ and $(Q, V, j, \beta)<(R, W, k, \gamma)$. Trivially the restriction $A_{R, W, k, \gamma} \rightarrow A_{S, X, l, \delta}$ for $(R, W, k, \gamma)<(S, X, l, \delta)$ carries $S_{R, W, k, \gamma}$ into $S_{S, X, l, \delta}$, and one can form a new antesheaf $\left\{S^{-1} A_{P, U, i, \alpha}, \bar{\rho}(Q, V, j, \beta ; P, U, i, \alpha)\right\}$ with $S^{-1} A_{P, U, i, \alpha}=$ $\left(S_{P, U, i, \alpha}\right)^{-1} A_{P, U, i, \alpha}$. The resulting sheaf is the quotient structure sheaf $\mathscr{S}^{-1} \mathscr{A}(M)$, whose algebra of global sections we denote $S^{-1} A(M)$.

DefinItion. For any fixed $m$, an antesheaf $\left\{F_{P, U, i, \alpha} \tau(Q, V, j, \beta ; P, U, i, \alpha)\right\}$ of free modules $F_{P, U, i, \alpha}$ of rank $m$ over $A_{P, U, i, \alpha}$ is regular if each $\operatorname{det} \tau(Q, V, j, \beta ; P, U, i, \alpha) \in A_{Q, V, j, \beta}$ belongs to $S_{Q, V, j, \beta} \subset A_{Q, V, j, \beta}$; in this 
case the resulting sheaf $\mathscr{F}$ over $\mathscr{A}(M)$ is a PL sheaf of "rank" $m$ (where "rank" does not imply that $\mathscr{F}$ is itself even locally free over $\mathscr{A}(M)$ ).

For any regular antesheaf one forms an antesheaf $\left\{S^{-1} F_{P, U, i, \alpha}\right.$, $\bar{\tau}(Q, V, j, \beta ; P, U, i, \alpha)\}$ in the obvious fashion with $S^{-1} F_{P, U, i, \alpha}=$ $\left(S_{P, U, i, \alpha}\right)^{-1} F_{P, U, i, \alpha}$, leading to a sheaf $\mathscr{S}^{-1} \mathscr{F}$ over $\mathscr{S}^{-1} \mathscr{A}(M)$.

Proposition. For any PL sheaf $\mathscr{F}$ of "rank" $m$ over $\mathscr{A}(M)$, the sheaf $\mathscr{S}^{-1} \mathscr{F}$ is locally free of rank $m$ over $\mathscr{S}^{-1} \mathscr{A}(M)$ in the sense that each $P \in M$ possesses a neighborhood $U$ such that $\Gamma\left(U, \mathscr{S}^{-1} \mathscr{F}\right)$ is free of rank $m$ over $\Gamma\left(U, \mathscr{S}^{-1} \mathscr{A}(M)\right)$; conversely every locally free sheaf of rank $m$ over $\mathscr{S}^{-1} \mathscr{A}(M)$ is of the form $\mathscr{S}^{-1} \mathscr{F}$ for some PL sheaf $\mathscr{F}$ of "rank" $\mathrm{m}$.

For later convenience we regard two PL sheaves $\mathscr{F}$ and $\mathscr{G}$ as equivalent whenever $\mathscr{S}^{-1} \mathscr{F}$ and $\mathscr{S}^{-1} \mathscr{G}$ are the same "vector bundle" in the sense of the preceding proposition, and we let $[\mathscr{F}]$ denote the coset containing $\mathscr{F}$. One verifies that if $\mathscr{F}$ and $\mathscr{G}$ are equivalent on $M$ then their pullbacks along any PL map $\Xi: M^{\prime} \rightarrow M$ are equivalent on $M^{\prime}$. Hence the pullback $\Xi^{!}[\mathscr{F}]$ of $[\mathscr{F}]$ is well-defined, and the resulting fibered category is the category of $P L$ sheaves.

It may happen that a coset $[\mathscr{F}]$ contains a PL sheaf $\mathscr{G}$ which is itself locally free over the structure sheaf $\mathscr{A}(M)$. Such a $\mathscr{G}$ corresponds in an obvious way to a bona fide vector bundle over $M$, and one easily verifies that this correspondence provides an embedding of the bona fide vector bundles on PL manifolds as a full subcategory of the category of PL sheaves. For convenience we simply say that $[\mathscr{F}]$ contains a vector bundle $\xi$ whenever $[\mathscr{F}]$ contains such a $\mathscr{G}$.

3. Characteristic classes. If $M$ is an $n$-dimensional PL manifold then the sheaf $\mathscr{E}(M)$ of differential forms is a PL sheaf of "rank" $n$ over $\mathscr{A}(M)$, virtually by definition. The following lemma is less surprising than it appears if one first observes that $\varphi_{N}$ and its derivatives vanish much more rapidly as $x \rightarrow 0$ than any polynomial in $\varphi_{0}, \varphi_{1}, \ldots, \varphi_{N-1}$.

LEMMA. The canonical monomorphism $\mathscr{E}(M) \rightarrow \mathscr{S}^{-1} \mathscr{E}(M)$ over $\mathscr{A}(M)$ is an isomorphism.

This outrageous result permits one to compute the de Rham cohomology of $M$ for dimensions $>1$ in terms of a complex $\left(\Lambda_{S^{-1} A(M)} S^{-1} E(M), d\right)$ for $S^{-1} E(M)=\Gamma\left(M, \mathscr{S}^{-1} \mathscr{E}(M)\right)$; in fact this is essentially the earlier de Rham complex except in dimension 0.

Now since $\mathscr{S}^{-1} \mathscr{F}$ is locally free for any PL sheaf $\mathscr{F}$, one easily constructs connections $D: \Lambda S^{-1} E(M) \otimes S^{-1} F \rightarrow \Lambda S^{-1} E(M) \otimes S^{-1} F$ for $S^{-1} F=\Gamma\left(M, \mathscr{S}^{-1} \mathscr{F}\right)$, as well as "inner products" $S^{-1} F \otimes S^{-1} F$ $\rightarrow S^{-1} A(M)$, using partitions of unity with entries in $A(M)$. This is all 
one needs for a Chern-Weil construction of real characteristic classes. In the first of the following results we consider cosets [F्F] of PL sheaves over the complexifications $\mathscr{A}(M)_{C}$ of structure sheaves $\mathscr{A}(M)$.

THEOREM. One can assign real Chern classes on the category of (cosets of) complex PLsheaves, in such a way that the following axioms are satisfied:

(i) if $[\mathscr{F}]$ is a coset of complex PL sheaves of "rank" $m$ on a PL manifold $M$ then the total Chern class $c([\mathscr{F}])$ is an element $1+c_{1}([\mathscr{F}])+$ $\cdots+c_{m}([\mathscr{F}]) \in H^{2 *}(M ; R)$ with $c_{i}([\mathscr{F}]) \in H^{2 i}(M ; R)$;

(ii) $c\left(\Xi^{!}[\mathscr{F}]\right)=\Xi^{*} c([\mathscr{F}]) \in H^{2 *}\left(M^{\prime} ; R\right)$ for any $P L$ map $\Xi: M^{\prime} \rightarrow M$;

(iii) $c([\mathscr{F}] \oplus[\mathscr{G}])=c([\mathscr{F}]) \cup c([\mathscr{G}])$ for any cosets $[\mathscr{F}]$ and $[\mathscr{G}]$ over $M$; and

(iv) if $[\mathscr{F}]$ contains a (complex) vector bundle $\xi$ over $M$ then $c([\mathscr{F}])$ is the classical total Chern class $c(\xi) \in H^{2 *}(M ; R)$.

We remark that one can define real Pontrjagin classes of real PL sheaves in the expected fashion in terms of Chern classes of their complexifications. In particular, the Pontrjagin classes of $[\mathscr{E}(M)]$ are the real Pontrjagin classes of the underlying PL manifold $M$. (See [3].)

Real oriented PL sheaves are defined in the obvious fashion.

THEOREM. One can assign real Euler classes on the category of (cosets of) real oriented PL sheaves, in such a way that the following axioms are satisfied:

(i) if $[\mathscr{F}]$ is a coset of real oriented PL sheaves of "rank" $2 n$ on a PL manifold $M$ then the Euler class $e([\mathscr{F}])$ belongs to $H^{2 n}(M ; R)$;

(ii) $e\left(\Xi^{!}[\mathscr{F}]\right)=\Xi^{*} e([\mathscr{F}]) \in H^{2 n}\left(M^{\prime} ; R\right)$ for any $P L$ map $\Xi: M^{\prime} \rightarrow M$;

(iii) $e([\mathscr{F}] \oplus[\mathscr{G}])=e([\mathscr{F}]) \cup e([\mathscr{G}])$ for any cosets $[\mathscr{F}]$ and $[\mathscr{G}]$ over $M$; and

(iv) if [Fु contains a (real oriented) vector bundle $\xi$ over $M$ then $e([\mathscr{F}])$ is the classical Euler class $e(\xi) \in H^{2 n}(M ; R)$.

By way of application, we mention that the first of the preceding theorems provides the tools for formulating Riemann-Roch identities, although no such identities have actually been established as yet.

\section{REFERENCES}

1. H. Osborn, Function algebras and the de Rham theorem in PL, Bull. Amer. Math. Soc. 77 (1971), 386-391.

2. —_ Differential geometry in PL, (mimeographed notes), University of Illinois, 1972.

3. - Pontrjagin classes of $P L$ sheaves, Bull. Amer. Math. Soc. (to appear).

Department of Mathematics, University of Illinois, Urbana, Illinois 61801 